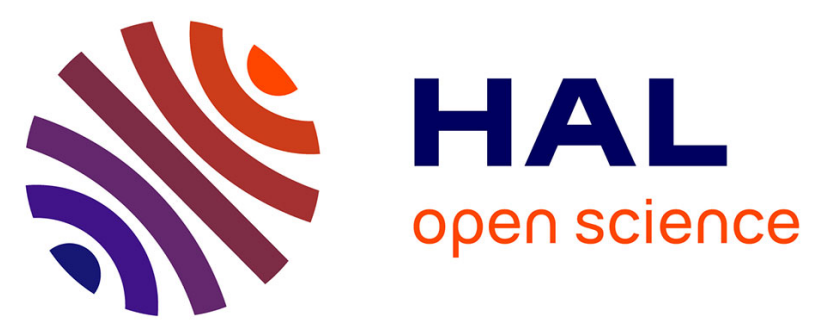

\title{
Dense arrays of microscopic optical vortex generators from femtosecond direct laser writing of radial birefringence in glass
}

\author{
Etienne Brasselet, Arnaud Royon, Lionel Canioni
}

\section{To cite this version:}

Etienne Brasselet, Arnaud Royon, Lionel Canioni. Dense arrays of microscopic optical vortex generators from femtosecond direct laser writing of radial birefringence in glass. Applied Physics Letters, 2012, 100 (18), pp.181901 (1-4). 10.1063/1.4705414 . hal-00682872

HAL Id: hal-00682872

https://hal.science/hal-00682872

Submitted on 27 Mar 2012

HAL is a multi-disciplinary open access archive for the deposit and dissemination of scientific research documents, whether they are published or not. The documents may come from teaching and research institutions in France or abroad, or from public or private research centers.
L'archive ouverte pluridisciplinaire HAL, est destinée au dépôt et à la diffusion de documents scientifiques de niveau recherche, publiés ou non, émanant des établissements d'enseignement et de recherche français ou étrangers, des laboratoires publics ou privés. 


\title{
Dense arrays of microscopic optical vortex generators from femtosecond direct laser writing of radial birefringence in glass
}

\author{
Etienne Brasselet, ${ }^{*}$ Arnaud Royon, ${ }^{\dagger}$ and Lionel Canioni ${ }^{\ddagger}$ \\ Univ. Bordeaux, LOMA, UMR 5798, F-33400 Talence, France. \\ CNRS, LOMA, UMR 5798, F-33400 Talence, France.
}

(Dated: March 23, 2012)

\begin{abstract}
We report on the generation of permanent singular light mode converters at the microscale using femtosecond direct laser writing in photo-thermo-refractive glass. It relies on the irreversible lightinduced radial birefringence in the bulk of the material. The ability of such birefringence pattern to convert the spin angular momentum of light into orbital optical angular momentum is exploited to demonstrate the production of large arrays of optical vortex generators with surface densities up to $10^{4} \mathrm{~cm}^{-2}$.
\end{abstract}

Femtosecond (fs) direct laser writing (DLW) is a well established technology that allows to fabricate threedimensional microstructures in transparent materials. It basically relies on light-induced controlled modifications of physical properties, for instance refractive index, absorption, luminescence or nonlinear optical characteristics, and it has applications in many fields, ranging from the technologies of information and telecommunications to biology and medicine [1].

In particular, fs-DLW has been found useful to fabricate singular micro-optical elements, namely structures enabling the generation of light fields endowed with phase and/or polarization singularities. An example is the realization of a photopolymerized microscopic spiral phase plate to control the orbital angular momentum imparted to optical tweezers [2]. Since then, fs-laser polymerization processes have matured and nowadays single and arrays of microscopic optical vortex generators with arbitrary topological charge can be achieved [3]. Another option relies on fs-DLW of space-variant birefringence pattern relying on form or true birefringence as demonstrated in fused silica [4] and sapphire [5], respectively. However, these approaches generally require the tedious point-bypoint definition of the singular optical elements.

Actually, the use of single-point fs-DLW has only been reported recently in glasses [6], whose principle of operation relies on the geometry of the damaged region rather than laser-induced birefringence. Still, the possibility to induce radial birefringence by single-point fsDLW in glass has also been reported [7]. Here we report on the single-point fs-DLW production of radial birefringence in a glassy material with an energy budget that is more than 100 times smaller than previously reported [7]. This is made possible by using photo-thermo-refractive (PTR) glass that has a similar composition as soda-lime glass, apart the presence of silver. Indeed the addition of photosensitive agents, such as silver, allows to enhance the light-induced material modifications while minimizing the energy budget [8]. Moreover we show that such a light-induced radial birefringence allows for the realization of microscopic optical vortex generators via a spinto-orbital optical angular momentum conversion process, which mimics the principle of operation of optical vortex generators based on radially ordered liquid crystal droplets [9]. Since the obtained singular light mode converters are permanently inscribed in glass at predetermined locations, dense arrays of them can be readily achieved and here we report on surface densities up to $10^{4} \mathrm{~cm}^{-2}$.

We used a 2 mm-thick slab of PTR glass with molar composition $\left\{70 \% \mathrm{SiO}_{2}, 15 \% \mathrm{Na}_{2} \mathrm{O}, 5 \% \mathrm{ZnO}, 4 \% \mathrm{Al}_{2} \mathrm{O}_{3}\right.$, $\left.5 \% \mathrm{NaF}, 1 \% \mathrm{KBr}, 0.01 \% \mathrm{Ag}_{2} \mathrm{O}\right\}$. It possesses an absorption cutoff wavelength at $260 \mathrm{~nm}$ (due to the absorption band associated with the silver ions) and a refractive in$\operatorname{dex} n=1.4959$ at $587.5 \mathrm{~nm}$ wavelength. This kind of glass has originally been developed for the recording of volume holograms [10]. It exhibits a negative refractive index change up to $6 \times 10^{-4}$ when exposed to continuouswave ultraviolet laser [11] or near-infrared femtosecond laser radiation $[12,13]$, both followed by thermal treatment. As mentioned above, note that our glass has no cerium in its composition. It has been shown that this glass, when exposed to near-infrared femtosecond laser pulses, exhibits the same response regarding to the presence or absence of cerium [13]. Indeed, in the case of femtosecond laser irradiation, the reduction of silver is initiated by the releasing of an electron from silver ions or the glass matrix, not from cerium ions [13].

The experimental setup is shown in Fig. 1(a). The sample is irradiated using a fs-laser source operating on the $\mathrm{TEM}_{00}$ mode with $M^{2}=1.2$ that emits 500 fs pulses at $1030 \mathrm{~nm}$ and $10 \mathrm{MHz}$ repetition rate. The maximum output average power is close to $6 \mathrm{~W}$, which gives a maximum energy of $600 \mathrm{~nJ}$ per pulse. The pulse energy is tuned by means of an acousto-optic modulator, which is also used as a controllable shutter. The sample is irradiated by the second-harmonic of the laser beam with wavelength $\lambda=515 \mathrm{~nm}$ that is produced using a frequency doubling crystal, a pair of lenses and a filter that blocks the fundamental harmonic. The laser beam is then focused using a microscope objective with numerical aperture $\mathrm{NA}=0.7$, the location of the beam focus is $d=150-200 \mu \mathrm{m}$ below the input facet of the sample and the beam waist diameter $2 w_{0}$ (defined at $\exp (-2)$ of its 
(a)
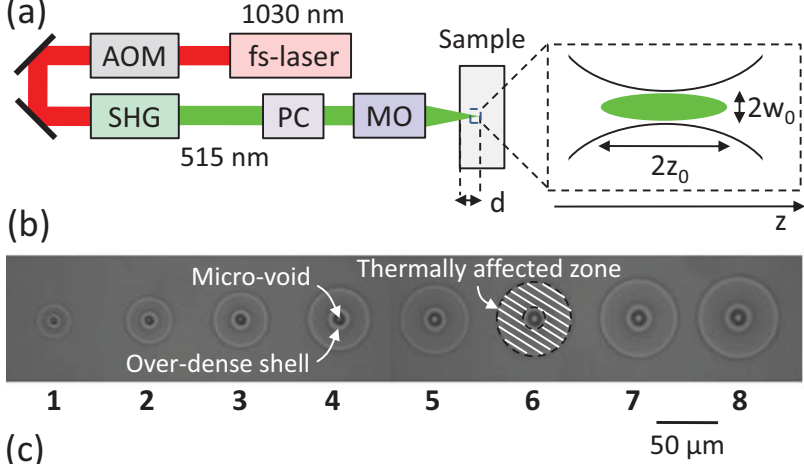

(c)

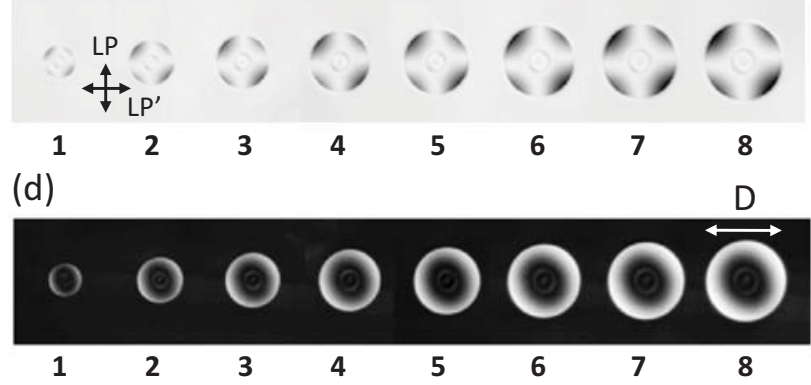

FIG. 1. (a) Sketch of the experimental set-up. AOM: acoustooptic modulator; SHG: second-harmonic generation stage; PC: polarization control stage; MO: microscope objective. The sample is a PTR glass whose composition is given in the text. (b) Unpolarized white light imaging of the photomodified glass using $N=10^{5}$ pulses and increasing fluence values $F=6.28,7.26,7.73,8.15,8.25,8.35,8.41$ and $8.46 \mathrm{~J} . c m^{-2}$ that refer to numbering from 1 to 8 , respectively. (c) Crossed linear polarization white light imaging with inverted grayscale, where LP and LP' refer to the direction of the linear polarizers. (d) Crossed circular polarization imaging at $532 \mathrm{~nm}$ wavelength. Here $d=150 \mu \mathrm{m}$.

maximal intensity) and the confocal parameter $2 z_{0}$, see Fig. 1(a), are estimated following $w_{0}=0.61 \lambda / \mathrm{NA}$ and $z_{0}=n \pi w_{0}^{2} / \lambda$, namely $2 w_{0}=0.9 \mu \mathrm{m}$ and $2 z_{0}=3.7 \mu \mathrm{m}$. In addition, half-wave and quarter-wave plates are inserted in the laser path to set the polarization state of the beam. The position of the sample is controlled using a three-dimensional translational stage with a $10 \mathrm{~nm}$ resolution.

By irradiating the sample with multi-shot exposure $\left(N=10^{5}\right.$ pulses $)$ we observe irreversible modifications that are visible under unpolarized white light imaging. This is shown in Fig. 1(b) where the labeling from 1 to 8 refers to fluence values $F=2 E /\left(\pi w_{0}^{2}\right)=6.28,7.26$, $7.73,8.15,8.25,8.35,8.41$ and $8.46 \mathrm{J.cm}^{-2}$, respectively, where $E$ is the average pulse energy. These modifications results from laser-induced refractive index changes due to the formation of $\mathrm{NaF}$ nanocrystallites around $\mathrm{Ag}^{0}$ nucleation centers [14]. The central part of the laser-induced structures consists in micro-voids surrounded by an overdense shell, as illustrated in Fig. 1(b). The formation mechanisms of such voids are well known in fused silica when using single [15] or multi-shot fs-excitation with a repetition period $T$ of the order of or shorter than the thermal diffusion characteristic time $\tau$ [16]. In our case $T=100 \mathrm{~ns}$ and, following the scaling $\tau \sim w_{0}^{2} / \mathcal{D}$ where $\mathcal{D}=0.46 \mu \mathrm{m}^{2} \mu \mathrm{s}^{-1}$ is the thermal diffusivity of the PTR glass, we get $T / \tau \sim 1$. This implies a temperature increase as a result of multi-shot exposure with the appearance of a thermally affected zone, see dashed region in Fig. 1(b), whose origin has been previously discussed in the particular case of soda-lime glass [7]. The appearance of a radial birefringence in the latter region is demonstrated from the observation of a well-defined dark cross pattern (note that the grayscale has been inverted for better readability) between crossed linear polarizers [see Fig. 1(c)] whatever the azimuthal position of the sample in the plane $(x, y)$ whereas a doughnut-shaped intensity profile is observed between crossed circular polarizers [see Fig. 1(d)]. Also, the results are found the same whatever the polarization state of the writing beam.

Towards a quantitative estimation of the lightinduced radial birefringence, the knowledge of the threedimensional geometry of the thermally affected zone is desirable. For this purpose we note that, when the irradiation starts, both the characteristic heat diffusion time along and perpendicular to the $z$ axis, namely $\tau_{\|} \sim z_{0}^{2} / \mathcal{D}$ and $\tau_{\perp} \sim w_{0}^{2} / \mathcal{D}$, which are of the order of $1 \mu \mathrm{s}$, are several orders of magnitude smaller than the time duration over which the glass is heated, namely $N T=10 \mathrm{~ms}$. At the end of the irradiation, the anisotropy of the heat source confined to the focal volume is therefore washed out and the resulting isotherm surfaces can be considered spherical, so as the thermally affected zone. This has been checked by solving numerically the threedimensional time-dependent heat equation with pulsed Gaussian heat source [7]. Then, for the sake of simplicity, the thermally affected zone is modeled as a cylinder of diameter $D$ with a volume identical to a sphere of diam-

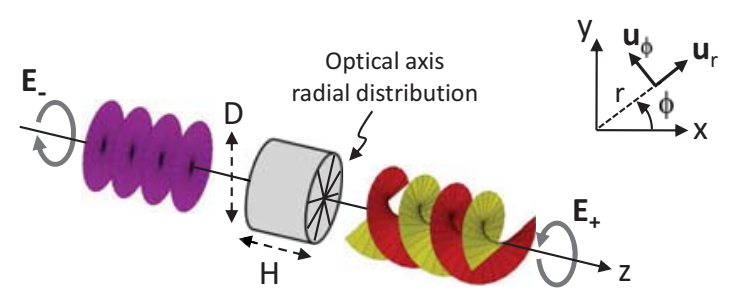

FIG. 2. Sketch of the optical vortex generation from spinto-orbital optical angular momentum conversion for the geometry used for the evaluation of the fs-laser-induced radial birefringence, where the photomodified region is assumed to be a cylinder of height $H$ and diameter $D$ with radial distribution of the optical axis. The input field is circularly polarized (here right-handed) and the output light field component whose polarization state is orthogonal to the input one carries a phase singularity with topological charge two, as illustrated by the equiphase surfaces. 
(a)
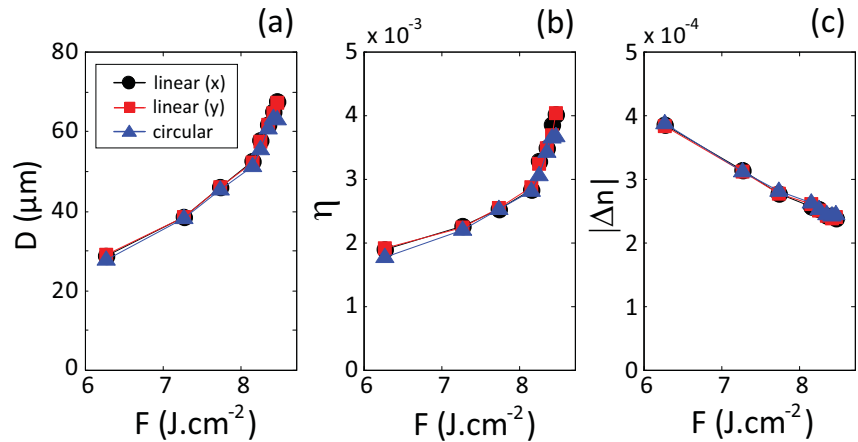

FIG. 3. Diameter $D$ of the thermally affected zone (a), spinto-orbital angular momentum conversion efficiency $\eta$ (b) and the estimated radial birefringence $\Delta n$ (c) [see text for details] as a function of the fluence. Data are shown for different polarization states of the writing beam as indicated in panel (a).

eter $D$, namely with height $H=2 D / 3$. We also assume a uniform radial birefringence $\Delta n$, which corresponds to the difference between the refractive indices along the radial and the azimuthal directions of a cylindrical coordinate system centered on the center of the irradiated region (see the corresponding unit vectors $\mathbf{u}_{r}$ and $\mathbf{u}_{\phi}$ on Fig. 2).

Then, neglecting diffraction effects, the output light field associated with a circularly polarized incident probe plane wave with electric field amplitude $E_{0}$ and wavelength in vacuum $\lambda_{\text {probe }}$ can be calculated using the Jones formalism. It turns out that the output field component whose polarization state is orthogonal to the incident one has, up to a phase factor, an amplitude $E_{0} \sin (\Phi / 2) \exp ( \pm 2 i \phi)$ where $\Phi=2 \pi H \Delta n / \lambda_{\text {probe }}$ is the overall optical phase delay experienced by probe light and $\phi$ the azimuthal angle in the $(x, y)$ plane with \pm sign referring to the handedness of the incident circular polarization state. The appearance of a phase singularity with topological charge two featured by the phase factor $\exp ( \pm 2 i \phi)$ is representative of the spin-to-orbital optical angular momentum process associated to radial birefringence, where the spin angular momentum flipping $( \pm \hbar \rightarrow \mp \hbar)$ related to polarization state changes is associated to the appearance of a $\pm 2 \hbar$ orbital angular momentum per photon. The latter process is illustrated in Fig. 2.

In a practical way, the diameter $D$ (hence $H$ ) is chosen from the well-defined size of the photomodified regions observed between crossed circular polarizers, as illustrated in Fig. 1(d) where $\lambda_{\text {probe }}=532 \mathrm{~nm}$. By doing so, we find the dependence of $D$ on the fluence that is shown in Fig. 3(a). On the other hand, the fraction of power of the incident probe light that is converted to the orthogonal circular polarization state, $\eta=\sin ^{2}(\Phi / 2)$, is measured from the ratio between the collected power over the disk of diameter $D$ under crossed circular po- larizers and the total output power over the same area, see Fig. 3(b). Finally, the radial birefringence is obtained following $|\Delta n|=\left(\lambda_{\text {probe }} / \pi H\right) \arcsin \sqrt{\eta}$ and we find $|\Delta n| \sim 3 \times 10^{-4}$ whatever is the polarization state of the writing beam, see Fig. 3(c). Note that, in view of the crude assumption of the proposed geometrical model, it is fair to say that the obtained data only aim at giving an order of magnitude of the radial birefringence.

The estimated value of the radial birefringence turns out to be of the same order of magnitude as the one measured in a previous work in soda-lime glass under multi-shot fs-DLW with $T / \tau \sim 1$ [7]. Indeed the radial birefringence data presented in Fig. 10 of Ref. [7] were obtained with $w_{0} \simeq 1.2 \mu \mathrm{m}(\lambda=800 \mathrm{~nm}$ and NA $=0.4)$ and $D \simeq 60 \mu \mathrm{m}$, hence $H \simeq 40 \mu \mathrm{m}$. Since the measured average retardance of an effective cylinder of height $H$ and diameter $D$ with uniform radial birefringence $\Delta n$ is estimated from Ref. [7] to be $|\Delta n| H \simeq 20 \mathrm{~nm}$, we obtain $|\Delta n| \sim 5 \times 10^{-4}$ in that case. Noteworthy, such a value is however obtain from $N=2.5 \times 10^{5}$ pulses with $2 \mu \mathrm{J}$ energy, namely a total input energy $\mathcal{E}=0.5 \mathrm{~J}[7]$. In contrast, in the present case, $N=10^{5}$ and the pulse energy is $E=15-20 \mathrm{~nJ}$, which gives $\mathcal{E}=N E=1.5-$ $2 \mathrm{~mJ}$. Therefore, we have lowered the energy budget by more than 100 times, which relies on the presence of a small amount of silver, namely $0.01 \%$ of $\mathrm{Ag}_{2} \mathrm{O}$ in our PTR glass, which enhances the light-induced refractive index change.

(a)
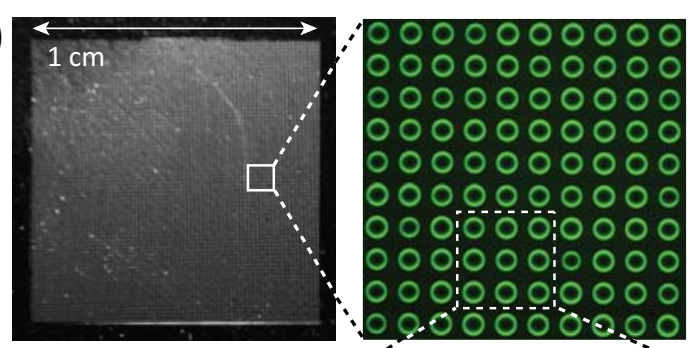

(d)
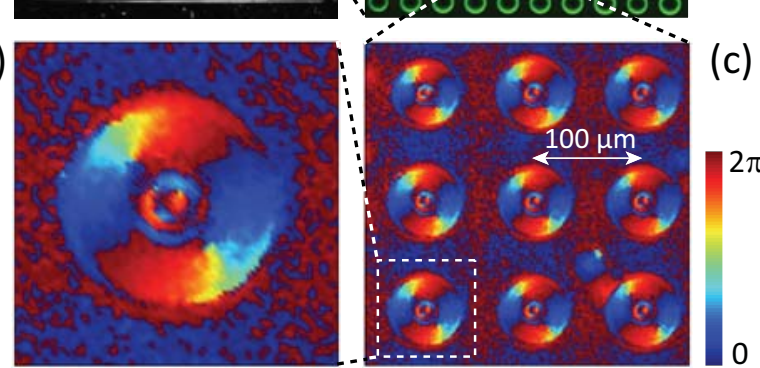

FIG. 4. (a) Array of $100 \times 100$ spin-to-orbital optical angular momentum converters over $1 \mathrm{~cm}^{2}$ area, each individual converters being obtained using $N=10^{5}$ pulses, a fluence $F=8.46 \mathrm{~J} . c m^{-2}$ and depth $d=200 \mu \mathrm{m}$. (b) Enlargement on $10 \times 10$ array of optical vortices obtained at the output of the sample at $532 \mathrm{~nm}$ wavelength. (c) Enlargement of panel (b) exhibiting the phase profile of the obtained optical vortices with topological charge two. (d) Zoom on the phase spatial distribution of a single optical vortex. 
Also, we note that laser-induced radial birefringence of a thermal origin has recently been reported in Ref. [17] in $\mathrm{Ag}^{+} / \mathrm{Na}^{+}$ion-exchanged soda-lime glass, however under different illumination conditions, namely continuouswave laser beam with much larger spot diameter of the order of $1 \mathrm{~mm}$. In addition, the light-induced retardance spatial profile is different too since the birefringence modifications extend far away the beam waist in that case [18]. Nevertheless, a quantitative comparison can be made by using the asymptotic value at large distance from the writing beam propagation axis of the expected radial birefringence, $\Delta n_{\infty}$. For this purpose, we extract from the data presented in Fig. 1 of Ref. [17] an asymptotic absolute phase delay $\Phi_{\infty}=45 \mathrm{rad}$, which gives $\left|\Delta n_{\infty}\right|=\left(\Phi_{\infty} / 2 \pi\right)\left(\lambda_{\text {probe }} / H\right) \sim 5 \times 10^{-3}$ for the used sample of thickness $H=0.85 \mathrm{~mm}$, where we took a typical probe beam wavelength in vacuum $\lambda_{\text {probe }}=633 \mathrm{~nm}$. Therefore a 10 times larger radial birefringence is found in Ref. [17], however with a total input energy $\mathcal{E}=70 \mathrm{~J}$ that is more that $10^{4}$ times larger than in the present case.

Moreover, despite a modest value for the spinto-orbital angular momentum conversion efficiency $\eta$, namely less than $1 \%$ as shown in Fig. 3(b), we stress that we obtain a rather good figure of merit that we define as $\xi=\eta / \mathcal{E}$. Indeed, here we obtain $\xi \sim 1 \mathrm{~J}^{-1}\left(\eta \simeq 3 \times 10^{-3}\right.$ and $\mathcal{E} \simeq 2 \mathrm{~mJ}$ ), which is typically 1000 times larger than the value $\xi \sim 10^{-3} \mathrm{~J}^{-1}$ (with $\eta \simeq 0.1$ and $\mathcal{E} \simeq 70 \mathrm{~J}$ ) reported in Ref. [17], where the spin-to-orbital angular momentum conversion has also been investigated.

Finally, we note that the ability to generate light beams endowed with phase singularities (i.e. optical vortex beams) at predetermined location and at the microscopic scale has been achieved here. This allows generating dense arrays of optical vortices, as illustrated in Fig. 4 where an array of $100 \times 100$ spin-to-orbital optical angular momentum converters over $1 \mathrm{~cm}^{2}$ area is shown in panel (a). Indeed, a characteristic doughnut-shaped light intensity profile is observed at the output of each individual converter under crossed circular polarizers, see Fig. 4(b). Moreover, the associated phase profiles all exhibit the expected winding of the phase by $4 \pi$ around the center of the beam, as shown in Fig. 4(c) and 4(d), where the phase spatial distribution is experimentally retrieved from spatially resolved polarimetric analysis following the procedure detailed in Ref. [9].

Noticeably, the obtained $10^{4} \mathrm{~cm}^{-2}$ surface densities of optical vortex generators is as dense as the one re- ported in [3] but extends over a thousand times larger area, which is made easily possible since the present direct laser writing does not need a point-by-point definition of each individual spin-to-orbital optical angular momentum converter.

The authors are grateful to J. Lumeau and L. B. Glebov for providing the photo-thermo-refractive glass.

* e.brasselet@loma.u-bordeaux1.fr

† a.royon@loma.u-bordeaux1.fr

¥ lionel.canioni@u-bordeaux1.fr

[1] R. R. Gattass and E. Mazur, Nature Photon. 2, 219 (2008).

[2] G. Knöner, S. Parkin, T. A. Nieminen, V. L. Y. Loke, N. R. Heckenberg, and H. Rubinsztein-Dunlop, Opt. Express 15, 5521 (2007).

[3] E. Brasselet, M. Malinauskas, A. Žukauskas, and S. Juodkazis, Appl. Phys. Lett. 97, 211108 (2010).

[4] M. Beresna, M. Gecevicius, P. G. Kazansky, and T. Gertus, Appl. Phys. Lett. 98, 201101 (2011).

[5] J. Morikawa, A. Orie, T. Hashimoto, and S. Juodkazis, Opt. Express 18, 8300 (2010).

[6] M. Beresna, M. Gecevicius, N. M. Bulgakova, and P. G. Kazansky, Opt. Express 19, 18989 (2011).

[7] M. Shimizu, M. Sakakura, M. Ohnishi, Y. Shimotsuma, T. Nakaya, K. Miura, and K. Hirao, J. Appl. Phys. 108, 073533 (2010).

[8] A. Royon, Y. Petit, G. Papon, M. Richardson, and L. Canioni, Opt. Mater. Express 1, 866 (2011).

[9] E. Brasselet, N. Murazawa, H. Misawa, and S. Juodkazis, Phys. Rev. Lett. 103, 103903 (2009).

[10] O. M. Efimov, L. B. Glebov, L. N. Glebova, K. C. Richardson, and V. I. Smirnov, Appl. Opt. 38, 619 (1999).

[11] O. M. Efimov, L. B. Glebov, and H. P. Andre, Appl. Opt. 41, 1864 (2002).

[12] S. Juodkazis, Lith. J. Phys. 42, 119 (2002).

[13] L. A. Siiman, J. Lumeau, and L. B. Glebov, J. NonCryst. Solids 354, 4070 (2008).

[14] T. Cardinal, O. Efimov, H. Francois-Saint-Cyr, L. Glebov, L. Glebova, and V. Smirnov, J. Non-Cryst. Solids 325, 275 (2003).

[15] E. N. Glezer and E. Mazur, Appl. Phys. Lett. 71, 882 (1997).

[16] S. M. Eaton, H. Zhang, P. R. Herman, F. Yoshino, L. Shah, J. Bovatsek, and A. Y. Arai, Opt. Express 13, 4708 (2005).

[17] J. Mostafavi-Amjad, H. R. Khalesifard, S. Slussarenko, E. Karimi, L. Marrucci, and E. Santamato, Appl. Phys. Lett. 99, 011113 (2011).

[18] E. Khazanov, O. V. Kulagin, S. Yoshida, D. B. Tanner, and D. H. Reitze, IEEE J. Quantum Electron. 35, 1116 (1999). 